\title{
Analysis of Human and System Factors on Errors in ICD Coding with Electronic Discharge Summary System
}

\author{
Hitomi Yamada ${ }^{1}$, Shigeki Kuwata ${ }^{2}$, Keunsik Park ${ }^{1}$, and Kenji Ohata ${ }^{1}$ \\ ${ }^{1}$ Department of Medical Informatics, Osaka City University Hospital, \\ 1-5-7 Asahi-machi, Abeno, Osaka, 545-8586, Japan \\ ${ }^{2}$ Medical Informatics Division, Tottori University Hospital, \\ 36-1 Nishi-cho, Yonago, Tottori, 683-8504, Japan \\ \{hitomiyama, kspark, kohata\} @med.osaka-cu.ac.jp, \\ shig@med.tottori-u.ac.jp
}

\begin{abstract}
This study aimed at clarifying the issues of the system that needs to be improved in order to raise the precision of computer-assisted ICD-10 encoding, by analyzing the inconsistency between the codes registered by physician and encoded by experts in discharge summaries of the electronic patient record system. It was found that human related factors led to the major inconsistency at the chapter level of ICD-10. Substantial commitment of coding experts was considered practically important to correct careless mistakes in physician's entry and assign accurate codes to complicated diagnoses, while relatively small improvement of the system was thought feasible as countermeasures against the inconsistency caused by system related factors.
\end{abstract}

Keywords: Coding, Electronic Patient Records, Human Errors, ICD-10, System Errors.

\section{Introduction}

This study aimed at clarifying the issues of the system that needs to be improved in order to raise the precision of computer-assisted ICD-10 encoding, by analyzing the difference between the codes registered by physician (system coding) and encoded by experts (expert coding) in discharge summaries of the electronic patient record system. The authors here defined the difference as an error, on the assumption that the expert coding would always appropriately reflect the diagnoses described in the summaries. The errors were then scrutinized in terms of human and system factors that would cause them.

\section{Methods}

Of the cases for patients who were discharged from April 1 to 15, 2009 at a university-affiliated hospital with approximately 1,000 beds in an urban district in Japan, 210 cases were selected for which the discharge summaries were registered and completed upon onset of the study. A cording expert encoded a primary diagnosis 
using ICD-10 for each summary, followed by the comparison with computer-assisted coding by physicians. When the errors were found, the system user interfaces were in turn examined to detect the reasons why physicians wrongly encoded. Subsequently the relationship between the error factors and the degree of the error (difference) was evaluated.

The error factors were categorized into (1) human factors and (2) system factors with subcategories of 1a. mistake or infringement, 1b. lack of details and 1c. difficult tasks for human factors, and of 2a. incorrectly assigned codes, $2 \mathrm{~b}$. difficult search, 2c. misleading presentation and $2 \mathrm{~d}$. no entries for system factors. The degree of the error was classified according to the structure of ICD-10 coding that included 22 chapters (e.g., I, XV) containing subdivided blocks (e.g., A00-A09, J90-J94). In the study, inconsistency between the system coding and the expert coding at the chapter level was referred to as major inconsistency, at the block level as middle inconsistency and at more detailed level as minor inconsistency.

\section{Results}

The inconsistency was found in $32 \%$ of the cases. Human factors were found in $91 \%$ of major, $79 \%$ of middle and $81 \%$ of minor inconsistency. Human factors caused a large portion of the major inconsistency, specifically careless mistakes (1a. 13/29) and too intricate coding requiring experts (1c. 14/29). Ambiguous coding (1c.) tended to frequently cause the minor consistency. On the other hand, system factors were found in $22 \%$ of major, $74 \%$ of middle and $88 \%$ of minor inconsistency, causing less major inconsistency than human factors.

\section{Discussion and Conclusion}

Functions or measures to prevent the errors equipped with the electronic discharge summary system are highly expected. Complicated mechanisms, such as case-based reasoning, however, would be required to reduce the careless mistakes and intricate codes that tended to cause the major inconsistency. In reality, manual encoding by experts may be the easiest way to solve those issues, hopefully with a little assistance of the artificial intelligence. On the other hand, feasible countermeasures against the faults in keyword search and data presentation would be able to be prepared by relatively facile tactics, e.g., improvement of the data quality in master files in which theoretical associations between diagnoses and ICD-10 codes are incorporated.

The inconsistency between system coding and expert coding was examined to raise the precision of computer-assisted ICD-10 encoding. It was found that human related factors led to the major inconsistency at the chapter level of ICD-10. Substantial commitment of coding experts was considered practically important to correct careless mistakes in physician's entry and assign accurate codes to complicated diagnoses, while relatively small improvement of the system was thought feasible as countermeasures against the errors caused by system related factors. 